\title{
In Vivo Validation of Restored Chordal Biomechanics After Mitral Ring Annuloplasty in a Rare Ovine Case of Natural Chronic Functional Mitral Regurgitation
}

\author{
Hanjay Wang ${ }^{1,2,+(\mathbb{D}}$, Michael J. Paulsen ${ }^{1,+} \mathbb{(}^{-}$, Annabel M. Imbrie-Moore ${ }^{1,3}$, Yuko Tada ${ }^{2,4}(\mathbb{D}$, \\ Hunter Bergamasco ${ }^{1}$, Sam W. Baker ${ }^{5}$, Yasuhiro Shudo ${ }^{1,2}$, Michael Ma ${ }^{1}$ and Y. Joseph Woo ${ }^{1,2,6, *}$ \\ 1 Department of Cardiothoracic Surgery, Stanford University, Stanford, CA 94305, USA; \\ hanjay@stanford.edu (H.W.); mpaulsen@stanford.edu (M.J.P.); aimbrie@stanford.edu (A.M.I.-M.); \\ hbergamasco@stanfordhealthcare.org (H.B.); yshudo@stanford.edu (Y.S.); mma@stanford.edu (M.M.) \\ Stanford Cardiovascular Institute, Stanford University, Stanford, CA 94305, USA; ytada@stanford.edu \\ Department of Mechanical Engineering, Stanford University, Stanford, CA 94305, USA \\ Department of Cardiovascular Medicine, Stanford University, Stanford, CA 94305, USA \\ Department of Comparative Medicine, Stanford University, Stanford, CA 94305, USA; \\ sambaker@stanford.edu \\ 6 Department of Bioengineering, Stanford University, Stanford, CA 94305, USA \\ * Correspondence: joswoo@stanford.edu; Tel.: +1-650-725-3828 \\ + These authors have contributed equally to this manuscript.
}

Academic Editor: Andy Wessels

Received: 22 April 2020; Accepted: 13 May 2020; Published: 15 May 2020

\begin{abstract}
Mitral valve chordae tendineae forces are elevated in the setting of mitral regurgitation (MR). Ring annuloplasty is an essential component of surgical repair for MR, but whether chordal forces are reduced after mitral annuloplasty has never been validated in vivo. Here, we present an extremely rare ovine case of natural, severe chronic functional MR, in which we used force-sensing fiber Bragg grating neochordae to directly measure chordal forces in the baseline setting of severe MR, as well as after successful mitral ring annuloplasty repair. Overall, our report is the first to confirm in vivo that mitral ring annuloplasty reduces elevated chordae tendineae forces associated with chronic functional MR.
\end{abstract}

Keywords: mitral valve; regurgitation; annuloplasty; biomechanics; chordae tendineae

\section{Introduction}

Ring annuloplasty is an essential component of mitral valve repair for the correction of mitral regurgitation (MR). Previous ex vivo studies have demonstrated that chordae tendineae forces are significantly elevated under simulated conditions of functional MR due to annular dilation and loss of leaflet coaptation [1] and that mitral annuloplasty may reduce these elevated chordal forces [2]. Because chordal force distribution impacts valve performance [3], the extent to which chordal biomechanics are recovered postoperatively may affect the long-term durability and outcomes of mitral repair. However, the fundamental concept that chordal forces are reduced after mitral annuloplasty has not been validated in vivo, due to the absence of large animal models with natural chronic MR and the ethical impermissibility of performing biomechanics experiments in patients. Here, we present an extremely rare ovine case of natural, severe chronic functional MR, in which we tested the hypothesis that ring annuloplasty repair of functional MR reduces chordae tendineae forces. 


\section{Case Report}

A male Dorset sheep (6 months old, $40 \mathrm{~kg}$ ) was diagnosed on cardiac magnetic resonance imaging (MRI) with left atrial (LA), left ventricular (LV), and mitral annular dilation (Table 1), as well as prominent central MR (Figure 1A). The sheep was planned for a terminal procedure involving mitral annuloplasty repair on cardiopulmonary bypass (CPB) with biomechanical analysis of chordal forces. The experiment was performed in accordance with the United States National Institutes of Health "Guide for the Care and Use of Laboratory Animals" (8th Edition, 2011), with approval by the Institutional Animal Care and Use Committee at Stanford University. Our strategy for sedation, anesthesia, analgesia, anticoagulation, and cardioplegia in ovine cardiac surgery involving CPB was described previously [4].

Table 1. Left heart measurements by cardiac magnetic resonance imaging.

\begin{tabular}{ccc}
\hline Left Heart Measurements & MR Sheep & Healthy Sheep \\
\hline Left Atrium Diameter & $5.26 \mathrm{~cm}$ & $2.83 \pm 0.24 \mathrm{~cm}$ \\
Left Atrium Volume & $229.0 \mathrm{~mL}$ & $31.5 \pm 8.2 \mathrm{~mL}$ \\
Left Ventricle Diameter & $7.94 \mathrm{~cm}$ & $4.49 \pm 0.30 \mathrm{~cm}$ \\
Left Ventricle Volume & $368.9 \mathrm{~mL}$ & $103.6 \pm 17.9 \mathrm{~mL}$ \\
Mitral Annulus Diameter & $5.57 \mathrm{~cm}$ & $3.08 \pm 0.07 \mathrm{~cm}$ \\
Mitral Annulus Area & $24.3 \mathrm{~cm}^{2}$ & $6.7 \pm 1.5 \mathrm{~cm}^{2}$ \\
\hline
\end{tabular}

Measurements were recorded at end-diastole for a rare male sheep with natural severe functional mitral regurgitation (MR) and compared to three representative healthy male sheep with the same age and weight (data presented as mean \pm standard deviation). Diameter measurements are presented as the average diameters from the 2-chamber and 4-chamber views on cardiac magnetic resonance imaging.

The sheep was intubated, and a right thoracotomy was performed. A large $1.5 \mathrm{~cm}$ patent ductus arteriosus (PDA) was discovered, suggesting over-circulation as the etiology of LV dilation and functional MR. Retrospectively, the PDA could be identified on MRI, although dedicated sequences were not obtained (Figure 1B). The PDA was ligated and divided. Baseline transesophageal echocardiography (TEE) confirmed severe holosystolic MR (jet area $40.4 \%$ of total LA area, vena contracta $8.6 \mathrm{~mm}$, Figure 1C). CPB was initiated via central aortic and bicaval cannulation. The heart was arrested with Del Nido cardioplegia, and a left atriotomy was made. Inspection of the mitral valve revealed a dilated annulus with intact subvalvular apparatus.

One secondary chord to the anterior leaflet (AS) and two primary chordae, one each to the anterior (AP) and posterior leaflets (PP), were instrumented with custom-designed force-sensing fiber Bragg grating neochordae (FBGN) [5]. For each target chord, a long $14 \mathrm{G}$ needle was inserted into the papillary muscle tip along the same vector as the chord, exiting through the LV apex and left chest. The FBGN was passed retrograde through the needle into the LV and secured at its two ends to the target chord using 5-0 polytetrafluoroethylene sutures. The chord was cut between the sutures, transferring the load to the FBGN (Figure 1D). The LA was closed, the aortic clamp was removed, the heart resumed a normal rhythm, and CPB was weaned off. TEE confirmed no change in severe MR after FBGN placement (jet area $40.9 \%$, vena contracta $7.7 \mathrm{~mm}$ ). With stable hemodynamics off CPB (heart rate 80-100 beats/min, mean arterial pressure $65-70 \mathrm{mmHg}$, central venous pressure $4-6 \mathrm{mmHg}$ ), chordal forces were measured in the state of severe MR (Figure 1E). Peak forces in the AS, AP, and PP chordae were $2.14 \mathrm{~N}, 0.59 \mathrm{~N}$, and $0.21 \mathrm{~N}$, respectively. Systolic $\mathrm{dF} / \mathrm{dt}$ (i.e., the time derivative of force) for the AS, AP, and PP chordae were $1441.8 \mathrm{~N} / \mathrm{s}, 969.9 \mathrm{~N} / \mathrm{s}$, and $550.5 \mathrm{~N} / \mathrm{s}$, respectively.

Next, the heart was re-arrested on $\mathrm{CPB}$ and the mitral valve was repaired with a $28 \mathrm{~mm}$ Carpentier-Edwards Physio II ring (Edwards Lifesciences, Irvine, CA) (Figure 1F). Cross-clamp and CPB times for the entire procedure were 150 and $261 \mathrm{~min}$, respectively. Post-repair TEE confirmed no MR (Figure 1G). Controlling for the same hemodynamics above, post-repair peak forces in the AS, AP, and PP chordae were $0.85 \mathrm{~N}, 0.01 \mathrm{~N}$, and $0.08 \mathrm{~N}$, respectively (Figure $1 \mathrm{H}$ ). Systolic dF/dt for the AS, AP, 
and PP chordae were $54.2 \mathrm{~N} / \mathrm{s}, 1.4 \mathrm{~N} / \mathrm{s}$, and $3.8 \mathrm{~N} / \mathrm{s}$, respectively. At the conclusion of the experiment, the sheep was euthanized via intravenous potassium chloride.
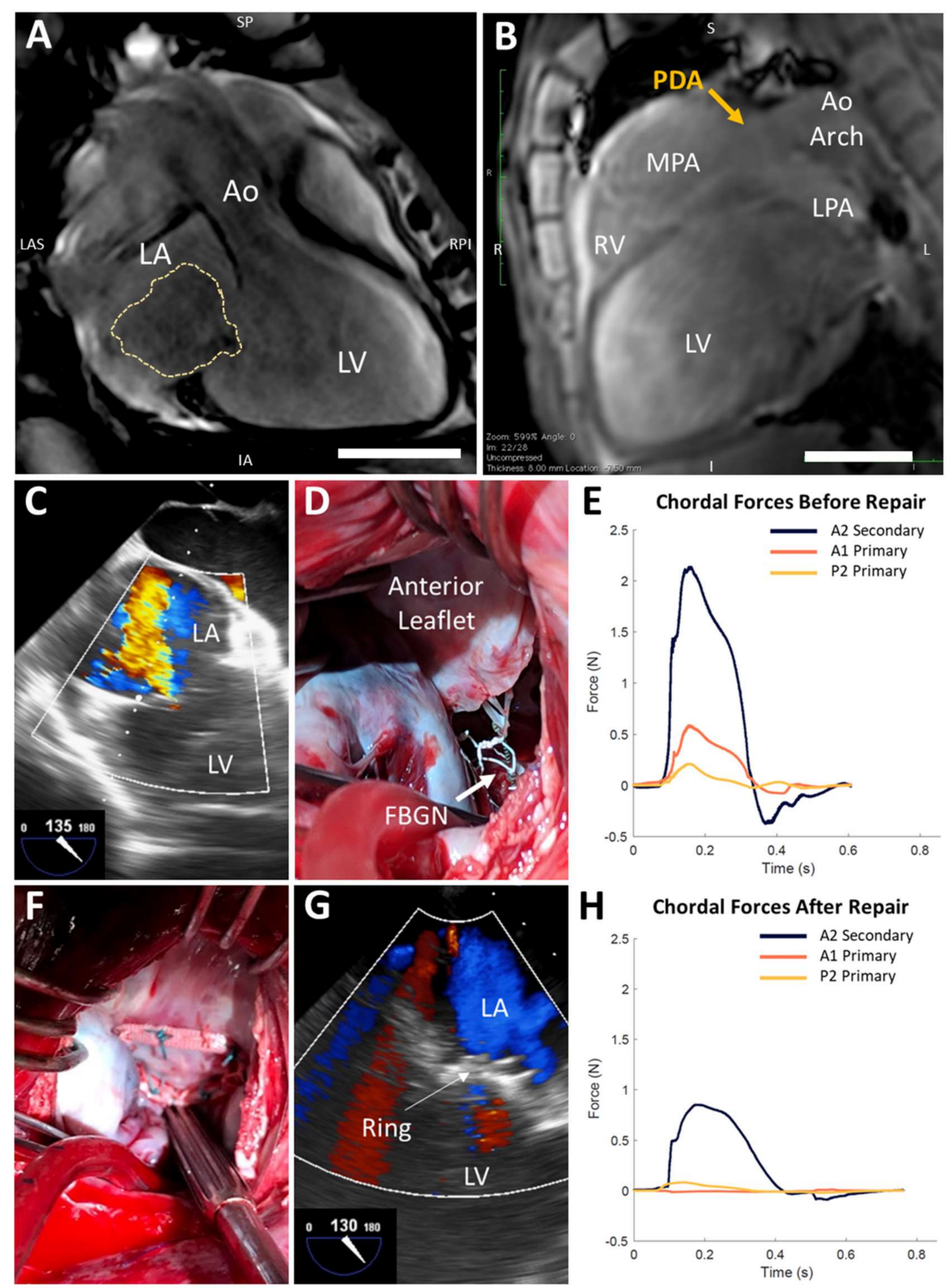

Figure 1. Chordal forces are reduced after mitral ring annuloplasty in a rare ovine case of natural severe functional mitral regurgitation. (A,B) Cardiac magnetic resonance imaging reveals left atrial (LA) and left ventricular (LV) dilation, central mitral regurgitation (MR, yellow trace), and a patent ductus arteriosus (PDA). Aorta (Ao); inferior (I); inferior-anterior (IA); left (L); left anterior-superior (LAS); left pulmonary artery (LPA); main pulmonary artery (MPA); right (R); right posterior-inferior (RPI); right ventricle (RV); superior (S); superior-posterior (SP). Scale bars, $5 \mathrm{~cm}$. (C) Baseline transesophageal echocardiography (TEE) confirms severe MR. (D) Instrumentation of native mitral valve chordae with force-sensing fiber Bragg grating neochordae (FBGN, white arrow). (E) Chordal forces in the state of severe MR over a representative cardiac cycle. (F) Mitral ring annuloplasty repair. (G) TEE confirms no MR after annuloplasty ring implantation. (H) Chordal forces are reduced after annuloplasty ring implantation. 


\section{Discussion}

Our report is the first to validate in vivo that mitral ring annuloplasty reduces elevated chordal forces associated with chronic functional MR. The in vivo forces we measured pre- and post-repair agreed well with previous ex vivo data assessing the impact of progressive annular dilation on chordal biomechanics [1].

Our unique ovine case involved severe chronic MR that developed naturally. Chronic left-to-right shunting through a large PDA resulted in persistent left heart volume overload, leading to gradual LA and LV dilation [6] and ultimately culminating in mitral annular dilation and development of functional MR. This pathophysiologic mechanism of functional MR has also been observed in human patients with PDA $[7,8]$.

Although we draw inferences from only one animal, due to its extreme rarity, ex vivo experiments are currently unable to precisely replicate the motion of the mitral annulus, papillary muscles, and left ventricle wall, all of which directly influence valvular biomechanics. Furthermore, chordal forces cannot be safely analyzed in vivo in human patients with mitral valve disease, and no reproducible in vivo large animal model of natural MR exists. Indeed, to our knowledge, a PDA has never previously been reported to result in severe MR in a sheep [9]. Although induced chronic MR models caused by chord cutting [10], LV-to-LA shunt creation [11], or myocardial infarction are available [12], our rare sheep with natural, severe functional MR precisely mirrors a clinical patient referral without prior manipulation of the mitral valve apparatus or LV, and therefore represents the most translationally-optimal opportunity to study the biomechanical foundation upon which mitral annuloplasty repair is based.

The use of FBGN in an in vivo setting carries some inherent limitations. For example, it is possible that, in the course of replacing the native chordae with FBGN sensors, we may have inadvertently altered the MR disease state from the baseline. However, TEE imaging provided quantitative evidence of persistently severe central MR after FBGN implantation. In addition, the forces we measured in the in vivo state of severe, chronic functional MR were relatively similar to those observed in a previous ex vivo MR model [1]. Furthermore, elimination of MR in vivo by ring annuloplasty repair resulted in a reduction of chordal forces to a level similar to that observed in a previous ex vivo mitral repair model [13]. These results of our in vivo experiment therefore support and build upon the existing ex vivo data describing mitral valve biomechanics.

Overall, we report the first in vivo biomechanical validation that mitral ring annuloplasty reduces abnormally elevated chordae tendineae forces associated with chronic functional MR. Future long-term studies including sheep with induced MR versus healthy controls are required to confirm our observations.

Author Contributions: Conceptualization, H.W., M.J.P., and Y.J.W.; methodology, H.W., M.J.P., A.M.I.-M., Y.T., H.B., S.W.B., Y.S., M.M., and Y.J.W.; formal analysis, H.W., M.J.P., A.M.I.-M., and Y.T.; investigation, H.W., M.J.P., A.M.I.-M., Y.T., H.B., S.W.B., Y.S., M.M., and Y.J.W.; resources, Y.J.W.; data curation, H.W., M.J.P., A.M.I.-M., and Y.T.; writing—original draft preparation, H.W. and M.J.P.; writing—review and editing, H.W., M.J.P., A.M.I.-M., Y.T., H.B., S.W.B., Y.S., M.M., and Y.J.W.; visualization, H.W., M.J.P., A.M.I.-M., and Y.T.; supervision, Y.J.W.; project administration, H.W., M.J.P., and Y.J.W.; funding acquisition, H.W., M.J.P., A.M.I.-M., and Y.J.W. All authors have read and agreed to the published version of the manuscript.

Funding: This study was funded in part by the National Institutes of Health (5R01HL089315-11, YJW), the American Heart Association (18POST33990223, HW; 17POST33410497, MJP), the National Science Foundation (GRFP DGE-1147470, AMI), and generous support from Donald and Sally O'Neal.

Acknowledgments: The authors thank the Stanford Cardiovascular Imaging Division for consultation regarding cardiac MRI interpretation, the Stanford University Veterinary Service Center for assistance with animal care, and members of Joseph Woo's laboratory (Amanda Steele, Lyndsay Stapleton, Daniel von Bornstädt, Kiah Williams, Anahita Eskandari, Justin Farry, Camille Hironaka, Kevin Jaatinen, Haley Lucian, and Akshara Thakore) for surgical support.

Conflicts of Interest: The authors declare no conflict of interest. The funders had no role in the design of the study; in the collection, analyses, or interpretation of data; in the writing of the manuscript; or in the decision to publish the results. 


\section{References}

1. Imbrie-Moore, A.M.; Paullin, C.C.; Paulsen, M.J.; Grady, F.; Wang, H.; Hironaka, C.E.; Farry, J.M.; Lucian, H.J.; Woo, Y.J. A novel 3D-Printed preferential posterior mitral annular dilation device delineates regurgitation onset threshold in an ex vivo heart simulator. Med Eng. Phys. 2020, 77, 10-18. [CrossRef] [PubMed]

2. Siefert, A.W.; Rabbah, J.-P.M.; Pierce, E.L.; Kunzelman, K.S.; Yoganathan, A.P. Quantitative Evaluation of Annuloplasty on Mitral Valve Chordae Tendineae Forces to Supplement Surgical Planning Model Development. Cardiovasc. Eng. Technol. 2014, 5, 35-43. [CrossRef] [PubMed]

3. Nielsen, S.L.; Nygaard, H.; Fontaine, A.A.; Hasenkam, J.; He, S.; Andersen, N.T.; Yoganathan, A.P. Chordal force distribution determines systolic mitral leaflet configuration and severity of functional mitral regurgitation. J. Am. Coll. Cardiol. 1999, 33, 843-853. [CrossRef]

4. Stapleton, L.M.; Steele, A.N.; Wang, H.; Hernandez, H.L.; Yu, A.C.; Paulsen, M.J.; Smith, A.A.A.; Roth, G.A.; Thakore, A.D.; Lucian, H.J.; et al. Use of a supramolecular polymeric hydrogel as an effective post-operative pericardial adhesion barrier. Nat. Biomed. Eng. 2019, 3, 611-620. [CrossRef] [PubMed]

5. Paulsen, M.; Bae, J.H.; Imbrie-Moore, A.M.; Wang, H.; Hironaka, C.; Farry, J.; Lucian, H.; Thakore, A.D.; Cutkosky, M.R.; Woo, Y.J. Development and Ex Vivo Validation of Novel Force-Sensing Neochordae for Measuring Chordae Tendineae Tension in the Mitral Valve Apparatus Using Optical Fibers With Embedded Bragg Gratings. J. Biomech. Eng. 2019, 142, 142. [CrossRef] [PubMed]

6. Schneider, D.J. The Patent Ductus Arteriosus in Term Infants, Children, and Adults. Semin. Perinatol. 2012, 36, 146-153. [CrossRef] [PubMed]

7. Wang, Z.; Chen, T.; Chen, L.; Qin, Y.; Zhao, X. Safety and Efficacy of Transcatheter Closure of Patent Ductus Arteriosus With Severe Mitral Regurgitation in Adults. J. Invasive Cardiol. 2016, 28, 30-33. [PubMed]

8. Kheiwa, A.; Ross, R.D.; Kobayashi, D. Reversal of severe mitral regurgitation by device closure of a large patent ductus arteriosus in a premature infant. Cardiol. Young 2016, 27, 189-192. [CrossRef] [PubMed]

9. Halleran, J.L.; Baumwart, R.D.; Dugat, D.R.; Streeter, R.N.; Phillips, H. Surgical ligation of patent ductus arteriosus in a dorper sheep. Veter. Surg. 2018, 48, 444-448. [CrossRef] [PubMed]

10. Shiota, T.; Jones, M.; Teien, D.E.; Yamada, I.; Passafini, A.; Ge, S.; Sahn, D.J. Dynamic change in mitral regurgitant orifice area: Comparison of color doppler echocardiographic and electromagnetic flowmeter-based methods in a chronic animal model. J. Am. Coll. Cardiol. 1995, 26, 528-536. [CrossRef]

11. Rankin, J.S.; Nicholas, L.M.; Kouchoukos, N.T. Experimental mitral regurgitation: Effects on left ventricular function before and after elimination of chronic regurgitation in the dog. J. Thorac. Cardiovasc. Surg. 1975, 70, 478-488. [CrossRef]

12. Gorman, J.H.; Gorman, R.C.; Jackson, B.M.; Enomoto, Y.; John-Sutton, M.G.S.; Edmunds, L.H. Annuloplasty ring selection for chronic ischemic mitral regurgitation: Lessons from the ovine model. Ann. Thorac. Surg. 2003, 76, 1556-1563. [CrossRef]

13. Paulsen, M.; Imbrie-Moore, A.M.; Wang, H.; Bae, J.H.; Hironaka, C.E.; Farry, J.; Lucian, H.J.; Thakore, A.D.; MacArthur, J.W.; Cutkosky, M.R.; et al. Mitral chordae tendineae force profile characterization using a posterior ventricular anchoring neochordal repair model for mitral regurgitation in a three-dimensional-printed ex vivo left heart simulator. Eur. J. Cardio-Thoracic Surg. 2019, 57, 535-544. [CrossRef] [PubMed]

(C) 2020 by the authors. Licensee MDPI, Basel, Switzerland. This article is an open access article distributed under the terms and conditions of the Creative Commons Attribution (CC BY) license (http://creativecommons.org/licenses/by/4.0/). 